\title{
HOMOLOGY 3-SPHERES BOUNDING ACYCLIC 4-MANIFOLDS
}

\author{
Y. Fukumoto And M. Furuta
}

\begin{abstract}
Let $\Sigma\left(a_{1}, a_{2}, \ldots, a_{n}\right)$ be a Seifert fibered homology 3-sphere with $a_{1}$ even. We show that if $\mu\left(\Sigma\left(a_{1}, a_{2}, \ldots, a_{n}\right)\right)=1 \bmod 2$, then the class of $\Sigma\left(a_{1}, a_{2}, \ldots, a_{n}\right)$ has infinite order in the homology cobordism group of homology 3-spheres. In the proof we use Seiberg-Witten's monopole equation on fourdimensional V-manifolds.
\end{abstract}

\section{Introduction}

Let $\Theta_{H}^{3}$ be the homology cobordims group of oriented homology 3-spheres. Then a class $[\Sigma] \in \Theta_{H}^{3}$ has infinite order if and only if the connected sum of any number of copies of $\Sigma$ cannot be the boundary of any acyclic 4-manifold. Our main theorem is:

Theorem 1. Let $\Sigma\left(a_{1}, a_{2}, \ldots, a_{n}\right)$ be a Seifert fibered homology 3-sphere. We assume that one of the $a_{i}$ 's is even. If $\mu\left(\Sigma\left(a_{1}, a_{2}, \ldots, a_{n}\right)\right)=1 \bmod 2$, then the homology cobordism class $\left[\Sigma\left(a_{1}, a_{2}, \ldots, a_{n}\right)\right] \in \Theta_{H}^{3}$ has infinite order.

R. Stern [14], S. Akbulut and R. Kirby [1], and A. J. Casson and J. L. Harer [2] constructed examples of Seifert fibered homology 3-spheres whose classes in $\Theta_{H}^{3}$ are zero.

The $\mu$-invariant gives a surjective homomorphism $\mu: \Theta_{H}^{3} \rightarrow \mathbf{Z}_{2}$. It implies that if $\mu(\Sigma)=1 \bmod 2$, then the order of the class $[\Sigma]$ is even or infinite. R. Fintushel and R. Stern [3] used the Donaldson theory on V-manifolds to obtain a sufficient condition for $\left[\Sigma\left(a_{1}, a_{2}, \ldots, a_{n}\right)\right]$ to have infinite order. Froyshov [5] constructed homomorphisms from $\Theta_{H}^{3}$ to $\mathbf{Z}$ by using the Donaldson-Floer theory. N. Saveliev [12] also obtained such a sufficient condition by making use of a theorem proved in [8] by using Seiberg-Witten theory.

In this note we use the Seiberg-Witten theory on V-manifolds to investigate Seifert fibered homology 3 -spheres.

We will define an integer $w\left(a_{1}, a_{2}, \ldots, a_{n} ; m\right)$ for pairwise-coprime positive integers $a_{1}, a_{2}, \ldots, a_{n}$ and for each integer $m$, by making use of index formula of some elliptic operators on 4 -V-manifolds.

The main theorem above follows from:

Received January 15, 1998.

Revision received May 25, 2000. 
Theorem 2. 1. If the class $\left[\Sigma\left(a_{1}, a_{2}, \ldots, a_{n}\right)\right]$ has finite order, then for any $m$ we have

$$
w\left(a_{1}, a_{2}, \ldots, a_{n} ; m\right) \leq 0
$$

The same conclusion holds when the connected sum of some number of copies of $\Sigma\left(a_{1}, a_{2}, \ldots, a_{n}\right)$ is the boundary of some positive definite 4manifold.

2. Suppose one of the $a_{i}$ 's is even. If the class $\left[\Sigma\left(a_{1}, a_{2}, \ldots, a_{n}\right)\right]$ has finite order, then

$$
w\left(a_{1}, a_{2}, \ldots, a_{n} ; s\right)=0, \quad \text { for } \quad s=\frac{1}{2}\left\{\alpha\left(2-\sum_{i=1}^{n}\left(1-\frac{1}{a_{i}}\right)\right)-1\right\} .
$$

When one of the $a_{i}$ 's is even, the integer $w\left(a_{1}, a_{2}, \ldots, a_{n} ; s\right)$ turns out to be an integral lift of the $\mu$-invariant of $\Sigma\left(a_{1}, a_{2}, \ldots, a_{n}\right)$.

The Casson invariant is an integral lift of the $\mu$-invariant. The above integer, however, is not equal to the Casson invariant in general.

In Section 2 we consider almost definite closed 4 - $\mathrm{V}$-manifolds by using monopole equation. In Section 3 we consider 4 - $\mathrm{V}$-manifolds with boundaries by using the results of the previous section. In Section 4 , we define some classes $\mathcal{S}\left(k^{+}, k^{-}\right)$ of oriented homology 3-spheres for non-negative integers $k^{+}$and $k^{-}$. The integral lift of $\mu$-invariant is constructed on $\mathcal{S}\left(k^{+}, k^{-}\right)$when $k^{+}$and $k_{-}$satisfy $k^{+}+k^{-} \leq 2$. In Section 5 we show that the Seifert fibered homology 3 -sphere $\Sigma\left(a_{1}, a_{2}, \ldots, a_{n}\right)$ is in the class $\mathcal{S}(0,1)$, if one of $a_{i}$ 's is even. We also give some examples.

\section{Closed V-4-manifolds}

We refer the reader to [11] for general properties of $\mathrm{V}$-manifold. Let $X$ be an oriented closed V-4-manifold. Let $b_{i}(X)$ be the $i$-th Betti number of $X$ and $b_{2}^{+}(X)$ (and $b_{2}^{-}(X)$ ) the dimension of maximal positive- (and negative-, respectively) definite subspace of $H^{2}(X, \mathbf{R})$.

We assume $b_{1}(X)=0$. Suppose a $\mathrm{V}$-spin ${ }^{c}$-structure on $X$ is given and fixed.

Fix a V-Riemannian metric on $X$. Let $S^{+}, S^{-}$be the associated positive and negative spinor bundles, and $L$ the associated V-line bundle $\wedge^{2} S^{+}=\wedge^{2} S^{-}$. From now on we omit the notation "V" in our terminology, since everything is defined in the category of $\mathrm{V}$-manifolds.

Let $D_{\mathrm{ASD}}(X)$ be the elliptic operator defined to be:

$$
D_{\mathrm{ASD}}(X)=d+d^{*}: \Omega^{0}(X) \oplus \Omega^{+}(X) \rightarrow \Omega^{1}(X),
$$

where $\Omega^{+}(X)$ is the space of self-dual 2-forms. For a fixed connection on $L$, we can define the Dirac operator

$$
D_{\text {Dirac }}(X): \Gamma\left(S^{+}\right) \rightarrow \Gamma\left(S^{-}\right)
$$

associated with the $\operatorname{spin}^{c}$-structure.

We denote by ind $D_{\mathrm{ASD}}(X)$ and ind $D_{\text {Dirac }}(X)$ the indices of these operators. Here the index is the difference between the real dimension of the kernel and that 
of the cokernel of the operator. Since the kernel and the cokernel of $D_{\text {Dirac }}(X)$ have complex structures, ind $D_{\text {Dirac }}$ is even. These indices can be calculated by using the index theorem for V-manifold due to T. Kawasaki [10]. The index of $D_{\text {ASD }}$ is, however, easily calculated by using harmonic V-forms and Hodge theory for $\mathrm{V}$-manifold. It is straightforward to extend the usual de Rham theorem to the $\mathrm{V}$-manifolds. This V-manifold version of de Rham theorem implies the following lemma.

Lemma 1. ind $D_{\mathrm{ASD}}(X)=1+b_{2}^{+}(X)$.

Here we used our assumption $b_{1}(X)=0$.

The formal dimension of the monopole moduli space for the $\operatorname{spin}^{c}$-structure is equal to ind $D_{\text {Dirac }}(X)$ - ind $D_{\mathrm{ASD}}(X)$. P. Kronheimer pointed out that for negative definite closed 4 -manifolds the formal dimension must be negative. The argument can be applied even for non-compact 4-manifolds as long as the monopole moduli space is compact [4]. We use $\mathrm{V}$-manifold version of this statement. Since $X$ is a closed V-manifold, the moduli space is compact. It implies:

Theorem 3. If $b_{2}^{+}(X)=0$, then we have ind $D_{\text {Dirac }} \leq 0$.

The following theorem is proved in [8] for smooth closed spin 4-manifolds. This proof can immediately be extended to closed spin V-4-manifolds as well.

Theorem 4. Suppose the spinc-structure is induced by a spin structure. Then ind $D_{\text {Dirac }}(X)$ is divisible by 4 . If ind $D_{\text {Dirac }}(X)$ is not zero, then we have

$$
1+\frac{1}{2} \text { ind } D_{\text {Dirac }}(X) \leq b_{2}^{+} \text {. }
$$

The right-hand side is equal to ind $D_{\mathrm{ASD}}(X)-1$ from Lemma 1 . In the proof of the above theorem we use this equality.

When the $\operatorname{spin}^{c}$-structure is induced by a spin structure, the kernel and the cokernel of $D_{\text {Dirac }}$ have the structure of vector space over quaternions, and hence ind $D_{\text {Dirac }}(X)$ is divisible by 4 .

Let $-X$ denote $X$ with reversed orientation. The spin structure of $X$ canonically induces a spin structure of $-X$. The corresponding Dirac operator is the formal adjoint of the original one. Hence we have ind $D_{\operatorname{Dirac}}(-X)=$ -ind $D_{\text {Dirac }}(X)$ and, under the assumption of the above theorem, we obtain:

Corollary 1. Suppose the spin ${ }^{c}$-structure is induced by a spin structure.

1. If ind $D_{\text {Dirac }}(X)$ is not zero, then we have

$$
1-\frac{1}{2} \text { ind } D_{\text {Dirac }}(X) \leq b_{2}^{-} \text {. }
$$

2. If $b_{2}^{+}(X), b_{2}^{-}(X) \leq 2$, then we have ind $D_{\text {Dirac }}=0$.

In particular, if $X$ is a spin 4 -V-manifold with $b_{2} \leq 2$, then ind $D_{\text {Dirac }}$ must be zero for the spin structure. 


\section{V-4-manifolds with boundaries}

In this section, suppose $X$ is a compact oriented V-4-manifold with boundary $\Sigma$. We assume that the singularities of $X$ are contained in its interior. Suppose $\Sigma$ is a homology 3 -sphere and $X$ has a $\operatorname{spin}^{c}$-structure $c$.

Let $Y$ be a spin 4-manifold with boundary $-\Sigma$. Since $H^{2}(\Sigma, \mathbf{Z})=0$, the $\operatorname{spin}^{c}$-structure on $\Sigma \times(0,1)$ is unique, and we have a $\operatorname{spin}^{c}$-structure on $X \cup_{\Sigma} Y$ by patching the $\operatorname{spin}^{c}$-structures on $X$ and $Y$. Moreover, since $H^{1}(\Sigma, \mathbf{Z})=0$, the patching is unique up to homotopy.

Definition 1. $w(\Sigma, X, c)$ is defined by:

$$
w(\Sigma, X, c):=\frac{1}{2} D_{\text {Dirac }}\left(X \cup_{\Sigma} Y\right)+\frac{1}{8}\left(b_{2}^{+}(Y)-b_{2}^{-}(Y)\right) .
$$

Note that each term of the right-hand side is an integer.

Lemma 2. The integer $w(\Sigma, X, c)$ is independent of the choice of $Y$ and its spin structure.

Proof. Since this kind of well-definedness is standard, we only give a sketch of the proof. Consider the elliptic operator $4 D_{\text {Dirac }} \oplus D_{\text {sign }}$, where $D_{\text {sign }}$ is the signature operator on $X \cup Y$, and the coefficient " 4 " indicates the direct sum of the four copies of $D_{\text {Dirac }}$. The Atiyah-Singer index theorem implies that the index of this operator vanishes for closed smooth 4-manifolds. Let $Y^{\prime}$ be another spin manifold with boundary $-\Sigma$. From an excision argument of the indices of the elliptic operators, we can show that the index for $X \cup_{\Sigma} Y$ is the sum of the indices of the operators for $X \cup_{\Sigma} Y^{\prime}$ and $\left(-Y^{\prime}\right) \cup_{\Sigma} Y$. (One explicit way to show this equality is to use Kawasaki's index theorem for $\mathrm{V}$-manifolds.) This implies that the indices for $X \cup_{\Sigma} Y$ and $X \cup_{\Sigma} Y^{\prime}$ are the same, from which the lemma immediately follows.

The same excision or localization argument also implies the following additivity.

Lemma 3. $w\left(\Sigma_{0} \sharp \Sigma_{1}, X_{0} \curvearrowleft X_{1}, c_{0} \downarrow c_{1}\right)=w\left(\Sigma_{0}, X_{0}, c_{0}\right)+w\left(\Sigma_{1}, X_{1}, c_{1}\right)$.

Here $\Sigma_{0} \sharp \Sigma_{1}$ is the connected sum of $\Sigma_{0}$ and $\Sigma_{1}$, and $X_{0} \downarrow X_{1}$ is the boundary connected sum of $X_{0}$ and $X_{1}$.

For $i=1,2, \ldots, m$, let $X_{i}$ be a compact oriented V-4-manifold with boundary $\Sigma_{i}$ such that its singularities are contained in its interiors. Suppose $\Sigma_{i}$ is a homology 3 -sphere and $X_{i}$ has a $\operatorname{spin}^{c}$-structure $c_{i}$ for each $i$.

From Theorem 3 and Lemma 3 we obtain:

Theorem 5. Suppose $X_{1}, X_{2}, \ldots, X_{m}$ are acyclic or negative definite. If the connected sum $\Sigma_{1} \sharp \Sigma_{2} \sharp \ldots \sharp \Sigma_{m}$ is the boundary of an acyclic 4-manifold, then we have

$$
\sum_{i=1}^{m} w\left(\Sigma_{i}, X_{i}, c_{i}\right) \leq 0
$$


Proof. Let $Y$ be the acyclic 4-manifold. Apply Theorem 3 to

$$
\left(X_{1} \curvearrowleft X_{2} \downarrow \ldots \downarrow X_{m}\right) \cup_{\Sigma_{1} \sharp \Sigma_{2} \sharp \ldots \sharp \Sigma_{m}}(-Y) \text {. }
$$

Before considering spin structure we note the following lemma.

Lemma 4. If $c$ is a spin structure of $X$, then we have

$$
\mu(\Sigma)=w(\Sigma, X, c) \bmod 2 .
$$

In particular, if $\Sigma$ is the boundary of an acyclic 4-manifold, then $w(\Sigma, X, c)$ must be even.

Proof. Since $\mu(\Sigma)=\left(b_{2}^{+}(Y)-b_{2}^{+}(Y)\right) / 8 \bmod 2$, it suffices to show that the index of $D_{\text {Dirac }}$ is divisible by 4 . This follows from the fact that the kernel and the cokernel of the Dirac operator has the structure of vector bundle over quaternions when the $\operatorname{spin}^{c}$-structure is indeced by a spin structure.

From Theorem 4 and Lemma 3, by using a similar argument as in the proof of Theorem 5, we obtain:

Theorem 6. Suppose all the $c_{i}$ 's are spin structures, and the connected sum $\Sigma_{1} \sharp \Sigma_{2} \sharp \ldots \sharp \Sigma_{m}$ is the boundary of an acyclic 4-manifold.

1. The sum $\sum_{i=1}^{m} w\left(\Sigma_{i}, X_{i}, c_{i}\right)$ is even.

2. If the above sum is not zero, then we have

$$
1-\sum_{i=1}^{m} b_{2}^{-}\left(X_{i}\right) \leq \sum_{i=1}^{m} w\left(\Sigma_{i}, X_{i}, c_{i}\right) \leq-1+\sum_{i=1}^{m} b_{2}^{+}\left(X_{i}\right) .
$$

Corollary 2. Suppose $c$ is a spin structure.

1. Suppose $b_{2}^{+}(X) \leq 2, b_{2}^{-}(X) \leq 2$ and $w(\Sigma, X, c) \neq 0$. Then the order of $[\Sigma] \in \Theta_{H}^{3}$ is even or infinite.

2. Suppose $b_{2}^{+}(X) \leq 1, b_{2}^{-}(X) \leq 1$ and $w(\Sigma, X, c) \neq 0$. Then the order of $[\Sigma] \in \Theta_{H}^{3}$ is infinite,

\section{An invariant for homology 3-spheres}

We introduce the following notation.

Definition 2. 1. Let $\mathcal{X}$ be the set of isomorphism classes of the triples $(\Sigma, X, c)$ that satisfy the following conditions.

(a) $X$ is a compact oriented spin $\mathrm{V}$-4-manifold such that its singularities lies in its interior.

(b) $\Sigma$ is the boundary of $X$. We assume that $\Sigma$ is a homology 3 -sphere.

(c) $c$ is a spin structure of $X$.

2. $\mathcal{X}\left(k^{+}, k^{-}\right)$is the subset of $\mathcal{X}$ given by

$$
\mathcal{X}\left(k^{+}, k^{-}\right):=\left\{(\Sigma, X, c) \in \mathcal{X} \mid b_{2}^{+}(X) \leq k^{+}, b_{2}^{-}(X) \leq k^{-}\right\} .
$$


3. $\mathcal{S}\left(k^{+}, k^{-}\right)$is the set of the isomorphism classes of oriented homology 3sphere $\Sigma$ such that $(\Sigma, X, c)$ is contained in $\mathcal{X}\left(k^{+}, k^{-}\right)$for some $X$ and $c$.

Note that the operation of connected sum induces the natural map:

$$
\mathcal{S}\left(k_{0}^{+}, k_{0}^{-}\right) \times \mathcal{S}\left(k_{1}^{+}, k_{1}^{-}\right) \rightarrow \mathcal{S}\left(k_{0}^{+}+k_{1}^{+}, k_{0}^{-}+k_{1}^{-}\right) .
$$

The next theorem is a corollary of Lemma 4 and Corollary 2.

Theorem 7. Suppose $\Sigma$ is in $\mathcal{S}(1,1)$. If $\mu(\Sigma)=1 \bmod 2$, then the order of $[\Sigma] \in \Theta_{H}^{3}$ is infinite.

Now we define an integral lift of $\mu$-invariant on $\mathcal{S}(2,0), \mathcal{S}(1,1)$ and $\mathcal{S}(0,2)$.

The invariant $w$ is defined on $\mathcal{X}$.

Theorem 8. Suppose $k^{+}$and $k^{-}$satisfy $k^{+}+k^{-} \leq 2$. Let $\left(\Sigma_{0}, X_{0}, c_{0}\right)$ and $\left(\Sigma_{1}, X_{1}, c_{1}\right)$ be two triples in $\mathcal{X}\left(k^{+}, k^{-}\right)$. Then we have

$$
w\left(\Sigma_{0}, X_{0}, c_{0}\right)=w\left(\Sigma_{1}, X_{1}, c_{1}\right) .
$$

Proof. Recall that $\Sigma_{0}$ and $\Sigma_{1}$ are homology cobordant to each other if and only if the connected sum of $\Sigma_{0}$ and $-\Sigma_{1}$ is the boundary of an acyclic 4-manifold. Since $X_{0} \downarrow-X_{1}$ satisfies the assumption of Corollary 2, we obtain

$$
w\left(\Sigma_{0}, X_{0}, c_{0}\right)+w\left(-\Sigma_{1},-X_{1},-c_{1}\right)=0 .
$$

In particular we have

$$
w\left(\Sigma_{1}, X_{1}, c_{1}\right)+w\left(-\Sigma_{1},-X_{1},-c_{1}\right)=0 .
$$

These two equality implies the required one.

The above theorem implies that the map $w$ descends to a map from $\mathcal{S}\left(k^{+}, k^{-}\right)$ to $\mathbf{Z}$ for $k^{+}$and $k^{-}$satisfying $k^{+}+k^{-} \leq 2$. We denote this map by $w\left(k^{+}, k^{-}\right)$:

$$
w\left(k^{+}, k^{-}\right): \mathcal{S}\left(k^{+}, k^{-}\right) \rightarrow \mathbf{Z} .
$$

Then the above theorem also implies:

Theorem 9. Suppose $k^{+}$and $k^{-}$satisfy $k^{+}+k^{-} \leq 2$. Then the map $w\left(k^{+}, k^{-}\right)$ is a homology cobordism invariant.

Remark. The authors do not know if the invariant is a homology cobordism invariant on the union of $\mathcal{S}(2,0), \mathcal{S}(1,1)$ and $\mathcal{S}(0,2)$.

The main theorem in this section is the following theorem, which is just a consequence of Lemma 4 and Corrollary 2

Theorem 10. Let $\Sigma$ be an oriented homology 3-sphere in $\mathcal{S}\left(k^{+}, k^{-}\right)$.

1. Suppose $k^{+}+k^{-} \leq 2$. Then the $\mu$-invariant $\mu(\Sigma)$ is given by:

$$
\mu(\Sigma)=w\left(k^{+}, k^{-}\right)(\Sigma) \bmod 2 .
$$

2. Suppose $k^{+}+k^{-} \leq 2$ and $w\left(k^{+}, k^{-}\right)(\Sigma) \neq 0$. Then the order of $[\Sigma] \in \Theta_{H}^{3}$ is even or infinite.

3. Suppose $k^{+}+k^{-} \leq 1$ and $w\left(k^{+}, k^{-}\right)(\Sigma) \neq 0$. Then the order of $[\Sigma] \in \Theta_{H}^{3}$ is infinite. 


\section{Seifert fibered homology 3-spheres}

In this section we give some examples of Seifert fibered homology 3-spheres, which are classified as follows.

Let $Z$ be a V-Riemann surface whose underlying space is $\mathbf{C} \mathbf{P}^{1}$ with $n$ marked points for $n \geq 3$. Let $a_{1}, a_{2}, \ldots, a_{n}$ be the order of the cyclic isotropy groups at the singular points. Assume that $a_{i}$ 's are pairwise coprime to each other. Then the abelian group consisting of all the isomorphism classes of V-line bundles is an infinite order cyclic group [9]. The first Chern class of one of the two generators is equal to $1 / \alpha$ for $\alpha=a_{1} a_{2} \ldots a_{n}$. Let $L_{0}$ be a $\mathrm{V}$-line bundle with $c_{1}\left(L_{0}\right)=-1 / \alpha$. Then other V-line bundles are of the form $L_{0}^{k}$ for some integer $k$.

We consider the disk bundle $X=D\left(L_{0}\right)$ as an oriented V-4-manifold. In general, when the isotropy representation at each singular point of $Z$ acts freely on the fiber of a V-line bundle $L$ except for the origin, the total space of the associated V-circle bundle $S(L)$ has natural smooth structure. The V-line bundle $L_{0}$ satisfies this condition. Hence we have a smooth oriented 3-manifold $S\left(L_{0}\right)=$ $\partial X$. We write $\Sigma\left(a_{1}, a_{2}, \ldots, a_{n}\right)$ for this oriented 3 -manifold. It is easy to check that this is a homology 3 -sphere, and that every oriented Seifert fibered homology 3 -sphere is of the form $\pm \Sigma\left(a_{1}, a_{2}, \ldots, a_{n}\right)$. See [9] for details. The canonical line bundle of the total space of $L_{0}$ is isomorphic to the dual of the pull-back of $L_{0} \otimes T Z$. Note that $T Z$ is isomorphic to $L_{0}^{k}$ for some $k$. Then the canonical line bundle is isomorphic to the pullback of $L_{0}^{-(k+1)}$.

The total space of $L_{0}$ has a canonical $\operatorname{spin}^{c}$-structure induced from its complex structure. As an open subset of $L_{0}$, we have a canonical $\operatorname{spin}^{c}$-structure of $X$.

Theorem 11. The V-4-manifold $X$ has a spin structure if and only if one of the $a_{i}$ 's is even.

Proof. Let $G_{n}$ be the subgroup of $U(n) \times U(1)$ consisting of $(g, z)$ satisfying $\operatorname{det} g=z^{2}$. Then $G_{n}$ is isomorphic to the fiber product of $U(n) \rightarrow S O(2 n)$ and $\operatorname{Spin}(2 n) \rightarrow S O(2 n)$. It implies that a complex manifold has a spin structure if and only if its canonical line bundle is a square of a line bundle. This argument holds for complex V-manifolds as well. Since the canonical line bundle of the total space of $L_{0}$ is isomorphic to the pullback of $L_{0}^{-(k+1)}$, the total space is spin if and only if $k$ is odd. We can calculate $k$ by looking at the Euler characteristic number of $Z[9]$ :

$$
k=\frac{\chi(Z)}{-1 / \alpha}=\alpha\left(-2+\sum_{i=1}^{n}\left(1-\frac{1}{a_{i}}\right)\right) .
$$

Since the right-hand side is odd if and only if one of the $a_{i}$ 's is even, the theorem follows.

Corollary 3. If one of the $a_{i}$ 's is even, then $\Sigma\left(a_{1}, a_{2}, \ldots, a_{n}\right)$ is in $\mathcal{S}(0,1)$. 
Let $T^{*}$ denote the complex cotangent bundle. The Dirac operator for the canonical $\operatorname{spin}^{c}$-structure is identified with the operator

$$
\bar{\partial}+\bar{\partial}^{*}: \Gamma\left(\wedge^{0,0} T^{*}\right) \oplus \Gamma\left(\wedge^{0,2} T^{*}\right) \rightarrow \Gamma\left(\wedge^{0,1} T^{*}\right) .
$$

The determinant of the spinor bundle for the canonical spin $^{c}$-structure is isomorphic to $\wedge^{2}\left(\wedge^{0,1} T^{*}\right) \cong \wedge^{2} T$, i.e., the dual of the canonical line bundle. We have to twist that spinor bundle by a line bundle to obtain the spinor bundle for the spin structure, Since the determinant of the spinor bundle for spin structure is trivial, the line bundle we have to use is the square root $L_{0}^{-(k+1) / 2}$ of the canonical line bundle.

Let $c(m)$ be the $\operatorname{spin}^{c}$-structure defined by the twisting of the canonical $\operatorname{spin}^{c}$ structure by $L_{0}^{m}$. Then the spin structure is given by $c((k+1) / 2)$.

Now the invariant $w\left(\Sigma\left(a_{1}, a_{2}, \ldots, a_{n}\right), X, c(m)\right)$ is calculated by using Vversion of the Atiyah-Singer index theorem [10]. The details of the arguments and also a comparison with other invariants of plumbing 3-manifolds are given in [6]. In this note we only state the result of calculation.

\section{Theorem 12.}

$$
\begin{aligned}
w\left(\Sigma\left(a_{1}, a_{2}, \ldots, a_{n}\right), X, c(m)\right)= & \frac{1}{8}\left[-\frac{1}{\alpha}\left\{\alpha\left(-2+\sum_{i=1}^{n}\left(1-\frac{1}{a_{i}}\right)\right)+1+2 m\right\}^{2}\right. \\
& +1-\sum_{i=1}^{n} \frac{1}{a_{i}} \sum_{l=1}^{a_{i}-1}\left\{\cot \left(\frac{\pi l}{a_{i}}\right) \cot \left(\frac{\pi b_{i} l}{a_{i}}\right)\right. \\
& \left.\left.+\cos \left(\frac{\pi\left(1+b_{i}+2 m b_{i}\right) l}{a_{i}}\right) \operatorname{cosec}\left(\frac{\pi l}{a_{i}}\right) \operatorname{cosec}\left(\frac{\pi b_{i} l}{a_{i}}\right)\right\}\right],
\end{aligned}
$$

where $b_{i}$ 's are integers which satisfy

$$
\sum_{i=1}^{n} b_{i} \frac{\alpha}{a_{i}}=-1
$$

In the introduction we used the notation $w\left(a_{1}, a_{2}, \ldots, a_{n} ; m\right)$ to denote this number.

Theorem 13. Suppose one of the $a_{i}$ 's is even. Then we have

$$
w\left(\Sigma\left(a_{1}, a_{2}, \ldots, a_{n}\right)\right)=w\left(a_{1}, a_{2}, \ldots, a_{n} ; s\right),
$$

for

$$
s=\frac{1}{2}\left\{\alpha\left(2-\sum_{i=1}^{n}\left(1-\frac{1}{a_{i}}\right)\right)-1\right\} .
$$

Here $w=w(1,1)$ or $w(0,2)$. 
More explicitly we have

$$
\begin{aligned}
w\left(\Sigma\left(a_{1}, a_{2}, \ldots, a_{n}\right)\right)=\frac{1}{8}\left[1-\sum_{i=1}^{n} \frac{1}{a_{i}} \sum_{l=1}^{a_{i}-1}\right. & \left\{\cot \left(\frac{\pi l}{a_{i}}\right) \cot \left(\frac{\pi b_{i} l}{a_{i}}\right)\right. \\
& \left.\left.+2 \epsilon_{i}^{l} \operatorname{cosec}\left(\frac{\pi l}{a_{i}}\right) \operatorname{cosec}\left(\frac{\pi b_{i} l}{a_{i}}\right)\right\}\right],
\end{aligned}
$$

where $\epsilon_{i}$ 's are defined by

$$
\epsilon_{i}=\left\{\begin{array}{lll}
(-1)^{1-b_{i}} & a_{i} \equiv 1 & (\bmod 2) \\
(-1)^{1-\Sigma_{j \neq i} b_{j}} & a_{i} \equiv 0 & (\bmod 2) .
\end{array}\right.
$$

We give some examples of Brieskorn homology 3-spheres such that the connected sum of any number of the copies cannot be the boundary of an acyclic 4-manifold. The following is a list of Brieskorn homology 3-spheres for which Theorem 5 is not applied, but Theorem 6 can be applied.

\begin{tabular}{|c|c|c|c|}
\hline Brieskorn & $\max \{w\}$ & spin case & Casson's invariant \\
\hline$\Sigma(2,3,7)$ & 0 & $w(-1)=-1$ & -1 \\
\hline$\Sigma(4,5,7)$ & 0 & $w(-29)=-1$ & -5 \\
\hline$\Sigma(7,9,10)$ & 0 & $w(-204)=-1$ & -25 \\
\hline$\Sigma(2,5,11)$ & 0 & $w(-12)=-1$ & -3 \\
\hline$\Sigma(5,8,11)$ & 0 & $w(-129)=-1$ & -17 \\
\hline$\Sigma(4,9,11)$ & 0 & $w(-109)=-1$ & -15 \\
\hline$\Sigma(5,7,12)$ & 0 & $w(-121)=-2$ & -16 \\
\hline$\Sigma(7,11,12)$ & 0 & $w(-316)=-1$ & -37 \\
\hline$\Sigma(3,4,13)$ & 0 & $w(-27)=-1$ & -5 \\
\hline$\Sigma(5,6,13)$ & 0 & $w(-109)=-1$ & -15 \\
\hline$\Sigma(3,8,13)$ & 0 & $w(-73)=-1$ & -11 \\
\hline
\end{tabular}

In the above list, $\Sigma(5,7,12)$ has the trivial $\mu$-invariant but non-trivial $w$ invariant.

Some other examples of plumbing type are given in [6].

Acknowledgments: The authors are grateful to Masaaki Ue and Vyacheslav S. Krushkal for pointing out some mistakes in the earlier version of the paper. The second author would like to thank the Max-Planck-Institut für Mathematik in Bonn for its hospitality.

\section{References}

[1] S. Akbulut and R. Kirby, Mazur manifolds, Michigan Math. J. 26 (1979), 259-284.

[2] A. J. Casson and J. L. Harer, Some homology lens spaces which bound rational homology balls, Pacific J. Math. 96 (1981), 23-36.

[3] R. Fintushel and R. Stern, Pseudofree orbifolds, Ann. of Math. 122 (1985), 335-364.

[4] K. A. Froyshov, The Seiberg-Witten equations and four manifolds with boundary, Math. Res. Lett. 3 (1996), 373-390.

[5] K. A. Froyshov, Equivariant aspects of Yang-Mills Floer theory, math. DG/9903083. 
[6] Y. Fukumoto, On an invariant of plumbed homology 3-spheres, preprint.

[7] M. Furuta, Homology cobordism group of homology 3-spheres, Invent. Math. 100 (1990), 339-355.

[8] M. Furuta, Monopole equation and the 11/8-conjecture, preprint.

[9] M. Furuta and B. Steer, Seifert fibred homology 3-spheres and the Yang Mills equations on Riemann surfaces with marked points, Adv. Math. 96 (1992), 38-102.

[10] T. Kawasaki, The index of elliptic operators over V-manifolds, Nagoya Math. J. 84 (1981), 135- 157.

[11] I. Satake, The Gauss-Bonnet theorem for V-manifolds, J. Math. Soc. Japan 9 (1957), 464-492.

[12] N. Saveliev, On the homology cobordism group of homology 3-spheres, Proc. Amer. Math. Soc. 126 (1998), 2819-2825.

[13] N. Saveliev, Floer homology of Brieskorn homology spheres: solution to Atiyah's problem, MSRI preprint 1997-6.

[14] R. Stern, Some more Brieskorn spheres which bound contractible manifolds, Notices Amer. Math. Soc. 25 (1978), A448.

Graduate School of Mathematical Sciences, University of Tokyo.

E-mail address: fukumoto@ms.u-tokyo.ac.jp

Graduate School of Mathematical Sciences, University of Tokyo.

E-mail address: furuta@ms.u-tokyo.ac.jp 